\title{
Racial/ethnic and sociodemographic factors associated with micronutrient intakes and inadequacies among pregnant women in an urban US population
}

\author{
Kelly J Brunst ${ }^{1, *}$, Robert $\bigcirc$ Wright ${ }^{1,2,3}$, Kimberly DiGioia ${ }^{4}$, Michelle Bosquet Enlow ${ }^{5,6}$, \\ Harriet Fernandez ${ }^{4}$, Rosalind J Wright ${ }^{1,2,3}{ }^{+}$and Srimathi Kannan ${ }^{7} \dagger$ \\ 'Kravis Children's Hospital, Department of Pediatrics, Icahn School of Medicine at Mount Sinai, One Gustave L. \\ Levy Place, Box 1198, New York, NY 10029, USA: ${ }^{2}$ Department of Preventive Medicine, Icahn School of \\ Medicine at Mount Sinai, New York, NY, USA: ${ }^{3}$ Mindich Child Health and Development Institute, Icahn School \\ of Medicine at Mount Sinai, New York, NY, USA: ${ }^{4}$ Channing Laboratory, Department of Medicine, Brigham and \\ Women's Hospital and Harvard School of Public Health, Boston, MA, USA: ${ }^{5}$ Department of Psychiatry, Boston \\ Children's Hospital, Boston, MA, USA: ${ }^{\circ}$ Department of Psychiatry, Harvard Medical School, Boston, MA, USA: \\ ${ }^{7}$ Department of Animal Science, Food and Nutrition College of Agricultural Sciences, Southern Illinois University, \\ Carbondale, IL, USA
}

Submitted 6 June 2013: Final revision received 26 October 2013: Accepted 27 0ctober 2013: First published online 13 December 2013

\begin{abstract}
Objective: To assess sociodemographic correlates of micronutrient intakes from food and dietary supplements in an urban, ethnically diverse sample of pregnant women in the USA.

Design: Cross-sectional analyses of data collected using a validated semi-quantitative FFQ. Associations between racial, ethnic and sociodemographic factors and micronutrient intakes were examined using logistic regression controlling for pre-pregnancy BMI, maternal age and smoking status.

Setting: Prenatal clinics, Boston, MA, USA.

Subjects: Analyses included pregnant women ( $n$ 274) in the PRogramming of Intergenerational Stress Mechanisms (PRISM) study, an urban longitudinal cohort designed to examine how stress influences respiratory health in children when controlling for other environmental exposures (chemical stressors, nutrition).

Results: High frequencies of vitamin E (52\%), Mg (38\%), Fe (57\%) and vitamin D (77\%) inadequacies as well as suboptimal intakes of choline (95\%) and K (99\%) were observed. Factors associated with multiple antioxidant inadequacies included being Hispanic or African American, lower education and self-reported economicrelated food insecurity. Hispanics had a higher prevalence of multiple methyl-nutrient inadequacies compared with African Americans; both had suboptimal betaine intakes and higher odds for vitamin $\mathrm{B}_{6}$ and $\mathrm{Fe}$ inadequacies compared with Caucasians. Nearly all women (98\%) reported $\mathrm{Na}$ intakes above the tolerable upper limit; excessive intakes of $\mathrm{Mg}$ (35\%), folate (37\%) and niacin (38\%) were also observed. Women reporting excessive intakes of these nutrients were more likely Caucasian or Hispanic, more highly educated, US-born and did not report food insecurity.

Conclusions: Racial/ethnic and other sociodemographic factors should be considered when tailoring periconceptional dietary interventions for urban ethnic women in the USA.
\end{abstract}

Suboptimal environmental conditions during critical periods of development, including inadequate nutrition, may programme physiological mechanisms and contribute to chronic disease risk ${ }^{(1)}$. Epidemiological research linking prenatal nutrition to children's health has focused largely on maternal macronutrient intakes such as energy and protein or correlates of nutritional status

$\dagger$ Co-senior authors. such as birth size and other indicators of fetal growth assessed retrospectively ${ }^{(2)}$. More recent evidence suggests the importance of specific micronutrients in prenatal programming of long-term health ${ }^{(3)}$. Moreover, various dietary components and inadequacies may mitigate or enhance the impact of chemical environmental exposures during early development as underscored by the National Advisory Environmental Health Sciences Council ${ }^{(4)}$. While epidemiological data are sparse for prenatal studies, 
dietary intake of antioxidants and methyl nutrients have been shown to reduce air pollution effects in non-pregnant adult samples ${ }^{(5,6)}$. Interactions have also been observed for other physical (e.g. tobacco smoke) and social (e.g. psychological stress) environmental exposures ${ }^{(7,8)}$. The methyl nutrient choline has recently been associated with alterations in the methylation state of genes influencing development of the fetal hypothalamic-pituitary-adrenal axis $^{(8)}$, which, in turn, has been linked to birth outcomes ${ }^{(9)}$ and respiratory and neurodevelopment during childhood ${ }^{(10)}$. Thus, understanding the correlates of inadequate dietary intakes may help inform effective intervention and prevention strategies in children's environmental health.

Studies from developing countries document dietary deficiencies in pregnancy as well as effects of micronutrient status on pregnancy outcomes ${ }^{(11)}$. A recent systematic review and meta-analysis demonstrates that pregnant women in developed countries, including the USA, are also at risk of suboptimal micronutrient intakes ${ }^{(12)}$. It is also unlikely that pregnant and childbearing-aged women in developed countries have adequate intake of micronutrients, such as $\mathrm{Fe}$ and $\mathrm{Ca}$, through foods alone ${ }^{(13)}$. Existing US studies have notable limitations, as most predate mandatory folic acid fortification, lack data on emerging essential micronutrients (i.e. choline, betaine) and few consider ethnic minorities. Of the three US studies including both African American and Hispanic women ${ }^{(14-10)}$, all examine a narrow range of micronutrients and lack assessments of choline and betaine intake, and only one considered both dietary and supplementation sources ${ }^{(12)}$.

Moreover, while studies in non-pregnant samples link a number of sociodemographic characteristics and dietary inadequacies, these associations have not been well studied in pregnant women in the USA. Minority status ${ }^{(17)}$, lower education ${ }^{(18,19)}$, food insecurity ${ }^{(20)}$ and lower income $^{(18)}$ have all been linked to poorer nutrition in non-pregnant US adult samples. Other studies suggest nativity (US-born $v$. foreign-born) ${ }^{(21)}$ and acculturation status (e.g. years living in the USA) ${ }^{(22)}$ also contribute to dietary behaviours, although such data are even more limited. This is an important area of research because health inequalities may be attributed, in part, to disparities in micronutrient intakes across race/ethnicity and socio-economic status (SES) ${ }^{(17)}$.

In order to begin to address some of these gaps, the objectives of the present study were twofold: (i) to comprehensively assess a wide range of dietary micronutrient inadequacies (including choline and betaine) from food and dietary supplements, which post-dates the mandatory folic acid fortification; and (ii) to examine the sociodemographic correlates of micronutrient intakes in an urban US population comprised largely of African American and Hispanic pregnant women. Classes of micronutrients examined in these analyses were selected based on our current understanding of the mechanisms through which nutritional factors in pregnancy may impact fetal growth and development and exert more long-term effects ${ }^{(23)}$.

\section{Experimental methods}

\section{Study population}

Participants were from the PRogramming of Intergenerational Stress Mechanisms (PRISM) study, a prospective pregnancy cohort of 276 mother-child pairs originally designed to examine how perinatal stress influences respiratory health in children when controlling for other environmental exposures (chemical stressors, nutrition). Women were recruited from prenatal clinics at 26.9 (sD 8.1) weeks' gestation from the Beth Israel Deaconess Medical Center and the East Boston Neighborhood Health Center from March 2011 to August 2012. Trained research staff approached women on selected clinic days. Recruitment sites were chosen given desired heterogeneity in sociodemographic and racial/ethnic characteristics. Eligibility criteria included: (i) English- or Spanish-speaking; (ii) age $\geq 18$ years at enrolment; and (iii) singleton pregnancy. All women approached to participate completed a screener questionnaire including data on key variables including maternal age, race/ ethnicity, education and nativity status. Procedures were approved by the human studies committee at the Brigham and Women's Hospital; written consent was obtained in the participant's primary language.

\section{Nutrition assessment and processing}

Maternal dietary intake was assessed at enrolment by trained interviewers using the modified Block98 (version 98.2; NutritionQuest, Berkeley, CA, USA) FFQ (Block 2006_Bodnar FFQ) consisting of approximately 120 food/beverage items ${ }^{(24,25)}$. The measure has been validated in multicultural populations ${ }^{(25,26)}$. Modifications were made to include a more extensive list of fish and seafood items ${ }^{(27)}$. The FFQ asks about usual food and beverage intakes in the 3 months prior to pregnancy; women were not asked to differentiate their intake before $v$. during pregnancy. The food list was developed from National Health and Nutrition Examination Survey (NHANES) III dietary recall data; portion size pictures enhanced accuracy of quantification. For each item, women were asked how often (daily, weekly, monthly or rarely/never) and how much (small, medium or large serving sizes) they consumed. The FFQ also queried the type and frequency of dietary supplements, especially vitamins and minerals, used in the prenatal period.

FFQ were processed through the online Block Dietary Data Systems (Berkeley, CA, USA) using the US Department of Agriculture (USDA) Food and Nutrient Database for Dietary Surveys (FNDDS) utilizing software originally developed at the National Cancer Institute ${ }^{(24)}$. Energy, macronutrient and micronutrient intakes were calculated per day by using nutrient values based on data from the 
third NHANES, the 1994-1996 Continuing Survey of Food Intakes by Individuals (CSFII) and the USDA Nutrient Database for Standard Reference ${ }^{(28)}$. Folate values were derived using the USDA 1998 nutrient database ${ }^{(28)}$. For foods with added folic acid, dietary folate equivalents (DFE) were calculated as: micrograms of naturally occurring food folate $+(\text { micrograms of added folic acid } \times 1 \cdot 7)^{(29)}$. Nutrient calculations were provided by NutritionQuest using the USDA Nutrient Database for Standard Reference ${ }^{(28)}$ for the dietary variables and related nutrients of interest. Betaine and choline values were derived from the USDA Database for the Choline Content of Common Foods ${ }^{(30)}$ and the USDA Database for the Choline Content of Common Foods, Release $2^{(31)}$. Unlisted values were imputed using recipes or applying values from similar foods.

Nutrient intakes were compared with the recommended nutrient intakes of the Institute of Medicine (Estimated Average Requirement (EAR), Adequate Intake (AI), Upper Tolerable Limit (UL)) when available ${ }^{(32-36)}$. Pregnancy energy cut-off values recommended by Meltzer et $a l^{(37)}$ and implemented by others ${ }^{(12)}$ were used. Women were considered at risk for nutrient inadequacy if their total (food + supplement) nutrient intake was less than the EAR or AI for pregnant women as done previously ${ }^{(25)}$. We calculated the likelihood of Fe inadequacy using the cut-point approach ${ }^{(34)}$.

\section{Maternal characteristics}

Information on race, maternal education, height and weight, smoking, nativity status and financial hardships was collected by interview-based questionnaire. Race/ ethnicity was self-reported and categorized as Caucasian, Hispanic, African American, or other/mixed. Nativity status was defined as being US-born or foreign-born. Acculturation was considered based on age at immigration, length of time living in the USA and primary language spoken (Spanish, English or bilingual). Maternal education was categorized as high (some college or college degree) or low (high school/high-school diploma or less than high school). Perceived financial difficulties were assessed by the self-reported degree of difficulty (no difficulty $v$. at least some degree of difficulty) in living on the total household income and meeting monthly payments/bills ${ }^{(38)}$; these items were addressed independently in the analyses. Women reported smoking (yes/no) at baseline and in the third trimester; women were classified as smokers if they reported smoking at either visit. Maternal BMI was calculated from height and pre-pregnancy weight reported at enrolment and categorized as normal weight (BMI $<25 \mathrm{~kg} / \mathrm{m}^{2}$ ), overweight (BMI $\geq 25$ to $<30 \mathrm{~kg} / \mathrm{m}^{2}$ ) or obese $\left(\mathrm{BMI} \geq 30 \mathrm{~kg} / \mathrm{m}^{2}\right)^{(39)}$.

\section{Ethnic identity}

Ethnic identity was ascertained using the fourteen-item subscale assessing positive ethnic attitudes and sense of belonging, ethnic identity achievement and ethnic behaviour, from the Multigroup Ethnic Identity Measure (MEIM) ${ }^{(40)}$.
The MEIM has been used in Caucasian, African American and Hispanic populations with good validity and reliability $^{(41)}$. Item responses, ranging from 1 (strongly disagree) to 4 (strongly agree), were summed (reverse scoring negatively worded items) and averaged over all items; the median split was used to index high and low ethnic identity scores.

\section{Economic-related food insecurity}

Two items assessed economic-related food insecurity: (i) 'How often do you not have enough money to afford the kind of food you and your family should have?' ('never', 'once in a while', 'often' and 'very often'; scores collapsed as never/ever), adapted from an economic strain measure $^{(38)}$; and (ii) 'In the past 6 months, did you go without food because you did not have the money to pay for it?' (yes/no), from the Crisis in Family Systems-Revised measure $^{(42)}$. Women responding 'ever' and/or 'yes', respectively, were considered to be experiencing food insecurity from an economic perspective.

\section{Statistical considerations}

Nutrient intakes were energy adjusted using the residual method $^{(43)}$. Because residuals have a mean of zero and include negative values, they do not provide an intuitive sense of intakes. Therefore, the predicted nutrient intake for the mean energy intake of the sample $(8354.6 \mathrm{~kJ}$ $(1996 \cdot 80 \mathrm{kcal})$ ) was used as a constant. Supplemental intakes were then added to the energy-adjusted nutrients to provide the total energy-adjusted intakes of each micronutrient.

Logistic regression was performed for each micronutrient, with inadequate intake as the outcome. All models were adjusted for maternal age (years), pre-pregnancy BMI and prenatal smoking status ${ }^{(44-46)}$. Micronutrient inadequacies resulting in low cell counts unable to generate reliable odds ratios were assessed by using the median of the micronutrient distribution as a cut-point to index high and low intakes. Due to the small number of bilingual women, analyses including primary language compare English- with Spanish-speaking women only. Results are presented as odds ratios and corresponding 95\% confidence intervals. The $\chi^{2}$ test was conducted to investigate possible differences in micronutrient intakes (dietary + supplements) above the $\mathrm{UL}^{(29,32-36)}$ by race, ethnic identity, food insecurity and other sociodemographic factors. Analyses assumed a two-sided alternative hypothesis, a 0.05 significance level and were conducted using the SAS statistical software package version $9 \cdot 2$.

\section{Results}

\section{Characteristics of respondents}

Of 389 eligible women approached, 276 agreed to participate $(70 \cdot 95 \%)$. There were no significant differences based on age, race/ethnicity, education and nativity 
Table 1 Participants' demographic and SES characteristics: pregnant women ( $n$ 274), PRISM study, Boston, MA, USA, March 2011-August 2012

\begin{tabular}{|c|c|c|}
\hline Characteristic & $n$ & $\%$ \\
\hline \multicolumn{3}{|l|}{ Race ( $n$ 274) } \\
\hline Hispanic & 116 & $42 \cdot 3$ \\
\hline African American & 48 & $17 \cdot 5$ \\
\hline Caucasian & 83 & $30 \cdot 3$ \\
\hline Other & 27 & $9 \cdot 9$ \\
\hline Low ethnic identity ( $n$ 266) & 144 & $54 \cdot 1$ \\
\hline \multicolumn{3}{|l|}{ Nativity status ( $n$ 272) } \\
\hline Foreign-born & 130 & $47 \cdot 8$ \\
\hline \multicolumn{3}{|l|}{ Primary language ( $n$ 250) } \\
\hline English & 159 & $63 \cdot 6$ \\
\hline Spanish & 78 & $31 \cdot 2$ \\
\hline Bilingual & 13 & $5 \cdot 2$ \\
\hline \multicolumn{3}{|l|}{ Financial hardship } \\
\hline \multicolumn{3}{|l|}{ Difficulty living on total household income (n 272) } \\
\hline No difficulty & 110 & $40 \cdot 4$ \\
\hline Somewhat difficult & 115 & $42 \cdot 3$ \\
\hline Difficult & 47 & $17 \cdot 3$ \\
\hline \multicolumn{3}{|l|}{ Difficulty meeting monthly payments (bills) ( $n$ 271) } \\
\hline No difficulty & 128 & $47 \cdot 2$ \\
\hline Somewhat difficult & 94 & $34 \cdot 7$ \\
\hline Difficult & 49 & $18 \cdot 1$ \\
\hline Evidence of food insecurity ( $n$ 266) & 74 & $27 \cdot 8$ \\
\hline \multicolumn{3}{|l|}{ Maternal education ( $n$ 266) } \\
\hline$<12$ th grade/HS degree or GED & 88 & $33 \cdot 1$ \\
\hline Some college/college degree (BA, MS, MD, PhD, etc.) & 178 & $66 \cdot 9$ \\
\hline \multicolumn{3}{|l|}{ Covariates } \\
\hline \multicolumn{3}{|l|}{ BMI status ( $n$ 236) } \\
\hline Normal weight $\left(\mathrm{BMI}<25 \mathrm{~kg} / \mathrm{m}^{2}\right)$ & 85 & $36 \cdot 0$ \\
\hline Overweight (BMI $\geq 25$ to $<30 \mathrm{~kg} / \mathrm{m}^{2}$ ) & 74 & $31 \cdot 4$ \\
\hline Obese $\left(\mathrm{BMI} \geq 30 \mathrm{~kg} / \mathrm{m}^{2}\right)$ & 77 & $32 \cdot 6$ \\
\hline \multirow[t]{2}{*}{ Smoked during pregnancy $(n$ 274) } & 46 & $16 \cdot 8$ \\
\hline & Mean & SD \\
\hline Maternal age (years) & $30 \cdot 36$ & $5 \cdot 64$ \\
\hline
\end{tabular}

PRISM, PRogramming of Intergenerational Stress Mechanisms; SES, socio-economic status; HS, high school; GED, General Educational Development; BA, Bachelor of Arts; MS, Masters; MD, Doctor of Medicine; PhD, Doctor of Philosophy.

status when comparing eligible women who participated and those who declined participation. Two women with implausible energy intake $(<2092 \mathrm{~kJ}(<500 \mathrm{kcal})$ or $>20920 \mathrm{~kJ}(>5000 \mathrm{kcal}))$ were excluded from analyses ${ }^{(37)}$, resulting in 274 women participating in the study. Table 1 summarizes sample characteristics. Women were predominantly minorities (42.3\% Hispanic, $17 \cdot 5 \%$ African American, $9 \cdot 9 \%$ other/mixed). More than half the sample reported a low ethnic identity $(54 \cdot 1 \%)$. Nearly half were foreign-born ( $47 \cdot 8 \%$ ), with the majority moving to the USA as adults $(67.7 \%)$ and residing in the USA for longer than 5 years $(78.5 \%)$. Most women identified as English-speaking (63.6\%), almost one-third identified as primarily Spanishspeaking $(31 \cdot 2 \%)$ and the remaining women identified as bilingual $(5 \cdot 2 \%)$. One-third reported a high school education or less. More than half identified difficulty living on total household income $(59 \cdot 6 \%)$ or meeting monthly payments $(52 \cdot 8 \%)$. Food insecurity was reported by over a quarter of women $(27 \cdot 8 \%)$. Additionally, the majority of women were overweight or obese $(64.0 \%)$, and $16.8 \%$ smoked during pregnancy. Age at time of immigration and duration in the USA were not associated with micronutrient inadequacies, and thus are not considered further.

\section{Intake of antioxidants}

Vitamin $\mathrm{E}$ and $\mathrm{Mg}$ were the most common inadequacies identified, with $51.5 \%$ and $38.3 \%$ of women consuming less than the EAR, respectively (Table 2). The prevalence of inadequacies of other antioxidants in descending order was Zn $(15 \cdot 3 \%)$, vitamin A $(10 \cdot 2 \%)$, vitamin C (7.3\%) and Se $(1 \cdot 1 \%)$. When compared with Caucasians, African Americans and Hispanics had greater odds of inadequate intakes across all antioxidants except $\mathrm{Mg}$ and $\mathrm{Zn}$ (Table 3). Low ethnic identity was associated with inadequate intakes of $\mathrm{Mg}$ (OR $=2 \cdot 19 ; 95 \%$ CI $1 \cdot 20,3 \cdot 99$ ). Being foreign-born was a significant predictor of vitamin $\mathrm{E}(\mathrm{OR}=3.73 ; 95 \%$ CI $2 \cdot 04,6 \cdot 82)$ and $\mathrm{Zn}(\mathrm{OR}=2 \cdot 51 ; 95 \%$ CI $1 \cdot 05,6 \cdot 00)$ inadequacies and $\beta$-carotene intakes below the median (OR $=2 \cdot 15 ; 95 \%$ CI $1 \cdot 22,3 \cdot 80)$. Spanish-speaking women were also more likely to report suboptimal intakes of $\beta$ carotene, vitamin E, Se and Zn compared with those women whose primary language was English (Table 3).

As shown in Table 4, low maternal education was most consistently associated with antioxidant inadequacies (vitamins $\mathrm{A}, \mathrm{C}$ and $\mathrm{E}, \boldsymbol{\beta}$-carotene, $\mathrm{Mg}, \mathrm{Zn}$ and $\mathrm{Se}$ ). Food insecurity was significantly associated with antioxidant inadequacies with the exception of $\beta$-carotene and $\mathrm{Zn}$. 
The odds of vitamin E inadequacy was increased more than twofold among those experiencing difficulty living on their total household income or difficulty in meeting monthly payments/bills. While overall $7 \cdot 3 \%$ of participants had vitamin $\mathrm{C}$ inadequacy, it was significantly more prevalent among those experiencing difficulty with living on their total household income (OR $=4 \cdot 20 ; 95 \%$ CI $1 \cdot 14$, 15.56) and meeting monthly payments/bills (OR $=4 \cdot 37$; $95 \%$ CI $1 \cdot 31,15 \cdot 50)$. This was also the case for suboptimal $\beta$-carotene intakes. Difficulty meeting monthly payments/bills was also a predictor of lower Se intakes. Women with intakes above the UL for Mg (35\%) were more likely to be Caucasian or Hispanic $\left(\chi^{2}=7 \cdot 96\right.$, $P=0 \cdot 05)$, more highly educated $\left(\chi^{2}=14 \cdot 77, P<0 \cdot 01\right)$ and less likely to report food insecurity $\left(\chi^{2}=10 \cdot 19\right.$, $P<0 \cdot 01)$ or difficulty living on their total household income $\left(\chi^{2}=3 \cdot 86, P=0 \cdot 05\right)$ or meeting payments/bills $\left(\chi^{2}=6 \cdot 02, P=0 \cdot 01\right)$. Women with vitamin A $(4 \cdot 7 \%)$, vitamin $\mathrm{C}(0 \cdot 36 \%)$, vitamin $\mathrm{E}(0 \%)$, Se $(0 \%)$ and $\mathrm{Zn}$ (1.8\%) intakes above the UL were infrequent.

\section{Methyl/epigenetic-nutrient intake}

The prevalence of Fe intakes below the EAR of $22-23 \mathrm{mg} /$ d was $56.6 \%$ (Table 2). Approximately $15 \%$ of women had folate and vitamin $\mathrm{B}_{6}$ inadequacies below the EAR. Inadequate choline intake was documented in almost all participants, with over $95 \%$ consuming less than the AI level. With respect to sociodemographic predictors of methyl-nutrient intakes, Hispanics had significantly lower intakes of vitamin $\mathrm{B}_{12}(\mathrm{OR}=2 \cdot 76 ; 95 \% \mathrm{CI} 1 \cdot 17,4 \cdot 43)$ and riboflavin $(\mathrm{OR}=3 \cdot 64 ; 95 \% \mathrm{CI} 1 \cdot 83,7 \cdot 22)$ compared with Caucasians; both African Americans and Hispanics had increased odds for inadequacies in vitamin $\mathrm{B}_{6}$ and $\mathrm{Fe}$ and suboptimal intakes of betaine compared with Caucasians (Table 3). Foreign-born women had greater odds of suboptimal intakes of vitamin $\mathrm{B}_{6}, \mathrm{Fe}$, methionine and betaine compared with those born in the USA. Spanishspeaking women also had greater odds of Fe, methionine and betaine suboptimal intakes compared with Englishspeaking women. Low ethnic identity was not associated with the intake of methyl/epigenetic nutrients.

Inadequacies in vitamin $\mathrm{B}_{6}(\mathrm{OR}=3 \cdot 39 ; 95 \% \mathrm{CI} 1 \cdot 51$, 7.62), $\mathrm{Fe}(\mathrm{OR}=3 \cdot 33 ; 95 \% \mathrm{CI} 1 \cdot 74,6 \cdot 39)$ and betaine $(\mathrm{OR}=7 \cdot 60 ; 95 \%$ CI $3 \cdot 80,15 \cdot 20)$ were significantly associated with lower education (Table 4 ). Fe inadequacy and low intake of betaine were significantly more common among women experiencing food insecurity or difficulty living on total household income or meeting monthly bills. Folate inadequacy was also significantly associated with difficulty living on the family's total income $(\mathrm{OR}=3 \cdot 22$; 95\% CI $1 \cdot 24,8 \cdot 35)$.

Women having intakes above the UL for folate $(n$ 102) were more likely to be Caucasian or Hispanic $\left(\chi^{2}=38 \cdot 65\right.$, $P<0 \cdot 01)$, US-born $\left(\chi^{2}=7 \cdot 27, P=0 \cdot 01\right)$, have a higher education $\left(\chi^{2}=24 \cdot 45, P<0 \cdot 01\right)$, and no report of food insecurity $\left(\chi^{2}=4 \cdot 88, P=0 \cdot 03\right)$ or difficulty living on their
Table 2 Frequencies of micronutrient inadequacies in the study participants: pregnant women ( $n$ 274), PRISM study, Boston, MA, USA, March 2011-August 2012

\begin{tabular}{|c|c|c|}
\hline Individual micronutrients ( $n$ 274) & $n$ & $\%$ \\
\hline \multicolumn{3}{|l|}{ Antioxidants } \\
\hline Vitamin A & 28 & $10 \cdot 2$ \\
\hline Vitamin C & 20 & $7 \cdot 3$ \\
\hline Vitamin E & 141 & $51 \cdot 5$ \\
\hline $\mathrm{Mg}$ & 105 & $38 \cdot 3$ \\
\hline $\mathrm{Se}$ & 3 & $1 \cdot 1$ \\
\hline $\mathrm{Zn}$ & 42 & $15 \cdot 3$ \\
\hline \multicolumn{3}{|l|}{ Methyl/epigenetic nutrients } \\
\hline Vitamin $\mathrm{B}_{12}$ & 8 & $2 \cdot 9$ \\
\hline Vitamin $\mathrm{B}_{6}$ & 40 & $14 \cdot 6$ \\
\hline Folate & 44 & $16 \cdot 1$ \\
\hline Riboflavin & 9 & $3 \cdot 3$ \\
\hline $\mathrm{Fe}$ & 155 & $56 \cdot 6$ \\
\hline Cholinetł & 261 & $95 \cdot 3$ \\
\hline \multicolumn{3}{|l|}{ Bone-related } \\
\hline Vitamin D & 211 & $77 \cdot 0$ \\
\hline $\mathrm{Ca}$ & 53 & $19 \cdot 3$ \\
\hline $\mathrm{Pt}$ & 3 & $1 \cdot 1$ \\
\hline \multicolumn{3}{|l|}{ Electrolytes } \\
\hline $\mathrm{K} t \ddagger$ & 273 & $99 \cdot 6$ \\
\hline Natł & 2 & $0 \cdot 7$ \\
\hline \multicolumn{3}{|l|}{ Other } \\
\hline Thiamin & 19 & $6 \cdot 9$ \\
\hline Niacin & 16 & $5 \cdot 8$ \\
\hline Vitamin $\mathrm{K} \ddagger \ddagger$ & 57 & $20 \cdot 8$ \\
\hline
\end{tabular}

PRISM, PRogramming of Intergenerational Stress Mechanisms. tBased on dietary intake only.

‡These nutrient intakes were compared with Adequate Intake (AI) levels; all other micronutrients were compared with the Estimated Average Requirement (EAR).

total family income $\left(\chi^{2}=10 \cdot 59, P<0 \cdot 01\right)$ or meeting payments/bills $\left(\chi^{2}=10 \cdot 36, \quad P<0 \cdot 01\right)$. Women with vitamin $\mathrm{B}_{6}(0 \%)$ and $\mathrm{Fe}(5 \cdot 8 \%)$ intakes above the UL were infrequent.

\section{Intake of bone-related micronutrients}

More than $75 \%$ of women had inadequate intakes of vitamin D (Table 2). Ca inadequacies were also prevalent (19.3\%). Only three women had P intake levels below the EAR. Being African American had a protective effect on vitamin D inadequacy $(\mathrm{OR}=0 \cdot 35 ; 95 \% \mathrm{CI} 0 \cdot 14,0 \cdot 87)$ and a negative effect on $\mathrm{Ca}$ inadequacy $(\mathrm{OR}=3 \cdot 15 ; 95 \%$ CI $1 \cdot 07,9 \cdot 27)$ compared with being Caucasian. Foreignborn $(\mathrm{OR}=2 \cdot 85$; $95 \%$ CI $1.45,5.59)$ and Spanishspeaking women $(\mathrm{OR}=4 \cdot 49 ; 95 \%$ CI $1 \cdot 78,11 \cdot 35)$ as well as those reporting low ethnic identity $(\mathrm{OR}=1 \cdot 93$; $95 \% \mathrm{CI}$ $1 \cdot 01,3 \cdot 68)$ had higher odds of vitamin $\mathrm{D}$ inadequacies (Table 3). Women with a low education level also had higher odds of vitamin D inadequacy (Table 4). Bone-related micronutrient intakes higher than the UL were not prevalent in this cohort (vitamin D (0\%), Ca (0.73\%), P (0\%)).

\section{Intake status of electrolytes}

With the exception of one participant, all women had $\mathrm{K}$ intakes below the AI level of $4.7 \mathrm{~g} / \mathrm{d}$ (mean intake of $2 \cdot 80 \mathrm{~g} / \mathrm{d}$ ). Thus, associations with race/ethnicity and SES factors were examined using the median to define high 
Table 3 Associations between race, ethnic identity and nativity and total (dietary + supplement) micronutrient inadequacy in the study participants: pregnant women (n 274), PRISM study, Boston, MA, USA, March 2011-August 2012

\begin{tabular}{|c|c|c|c|c|c|c|c|c|c|c|}
\hline \multirow[b]{2}{*}{ Micronutrients } & \multicolumn{2}{|c|}{ Foreign-bornt } & \multicolumn{2}{|c|}{ African American } & \multicolumn{2}{|c|}{ Hispanicł } & \multicolumn{2}{|c|}{ Spanish-speaking } & \multicolumn{2}{|c|}{ Low ethnic identity } \\
\hline & OR & $95 \% \mathrm{Cl}$ & OR & $95 \% \mathrm{Cl}$ & OR & $95 \% \mathrm{Cl}$ & OR & $95 \% \mathrm{Cl}$ & OR & $95 \% \mathrm{Cl}$ \\
\hline \multicolumn{11}{|l|}{ Antioxidants } \\
\hline Vitamin A & $1 \cdot 35$ & $0.51,3.56$ & $2 \cdot 75^{\star} \S$ & $1 \cdot 13,6 \cdot 68$ & $4 \cdot 79^{*} \S$ & $2 \cdot 37,9 \cdot 67$ & $1 \cdot 88$ & $0 \cdot 68,5 \cdot 21$ & $2 \cdot 02$ & $0.76,5 \cdot 41$ \\
\hline$\beta$-Carotene $\S$ & $2 \cdot 15^{\star}$ & $1 \cdot 22,3 \cdot 80$ & $3 \cdot 39^{*}$ & $1 \cdot 39,8 \cdot 26$ & $5 \cdot 75^{\star}$ & $2 \cdot 82,11 \cdot 73$ & $4 \cdot 01^{*}$ & $2 \cdot 12,7 \cdot 93$ & $1 \cdot 22$ & $0 \cdot 70,2 \cdot 14$ \\
\hline Vitamin C & $1 \cdot 34$ & $0 \cdot 47,3 \cdot 85$ & $8 \cdot 25^{\star}$ & $1 \cdot 36,49 \cdot 94$ & $7 \cdot 04^{\star}$ & $1 \cdot 40,35 \cdot 28$ & 1.06 & $0 \cdot 33,2 \cdot 63$ & $1 \cdot 43$ & $0.51,3.97$ \\
\hline Vitamin E & $3 \cdot 73^{\star}$ & $2 \cdot 04,6 \cdot 82$ & $2 \cdot 49^{\star}$ & $1.04,5.99$ & $4 \cdot 66^{\star}$ & $2 \cdot 33,9 \cdot 32$ & $5 \cdot 36^{\star}$ & $2 \cdot 71,10 \cdot 62$ & $1 \cdot 17$ & $0.66,2.08$ \\
\hline $\mathrm{Mg}$ & $1 \cdot 41$ & $0 \cdot 79,2 \cdot 50$ & $1 \cdot 23$ & $0.51,2.95$ & $1 \cdot 61$ & $0 \cdot 82,3 \cdot 17$ & 1.52 & $0 \cdot 81,2 \cdot 86$ & $2 \cdot 19$ & $1.20,3.99$ \\
\hline $\mathrm{Se} \S$ & $3 \cdot 80$ & $2 \cdot 12,6 \cdot 80$ & $5 \cdot 25^{\star}$ & $2 \cdot 64,10 \cdot 43$ & $4 \cdot 32^{*}$ & $1 \cdot 80,10 \cdot 36$ & $5 \cdot 12^{\star}$ & $2 \cdot 63,9.94$ & 0.85 & $0.49,1.46$ \\
\hline $\mathrm{Zn}$ & $2 \cdot 51^{*}$ & $1 \cdot 05,6 \cdot 00$ & $1 \cdot 52 \S$ & $0.64,3.63$ & $3 \cdot 39 * \S$ & $1 \cdot 72,6 \cdot 69$ & $3 \cdot 13^{*}$ & $1 \cdot 29,7 \cdot 61$ & $2 \cdot 29$ & $0.97,5.41$ \\
\hline \multicolumn{11}{|c|}{ Methyl/epigenetic nutrients } \\
\hline Vitamin $\mathrm{B}_{12}$ & 0.42 & $0 \cdot 08,2 \cdot 24$ & $0.62 \S$ & $0.25,1.50$ & $2 \cdot 76^{*} \S$ & $1 \cdot 17,4 \cdot 43$ & 0.82 & $0 \cdot 12,5 \cdot 64$ & 0.22 & $0.02,1.99$ \\
\hline Vitamin $\mathrm{B}_{6}$ & $2 \cdot 42^{\star}$ & $1 \cdot 04,5 \cdot 62$ & $4 \cdot 53^{\star}$ & $1 \cdot 06,19 \cdot 33$ & $5 \cdot 65^{\star}$ & $1 \cdot 55,20 \cdot 53$ & $2 \cdot 24$ & $0 \cdot 96,5 \cdot 24$ & $1 \cdot 84$ & $0.82,4 \cdot 17$ \\
\hline Folate & $0 \cdot 82$ & $0 \cdot 37,1 \cdot 82$ & $2 \cdot 65$ & $0.83,8 \cdot 42$ & 1.94 & $0.72,5 \cdot 23$ & 0.92 & $0.25,3.40$ & $1 \cdot 60$ & $0.73,3.53$ \\
\hline Riboflavin & $0 \cdot 86$ & $0 \cdot 17,4 \cdot 42$ & $2 \cdot 00 \S$ & $0.83,4.82$ & $3 \cdot 64 * \S$ & $1 \cdot 83,7 \cdot 22$ & $1 \cdot 31$ & $0 \cdot 23,7 \cdot 40$ & 0.58 & $0 \cdot 10,3 \cdot 32$ \\
\hline $\mathrm{Fe}$ & $3 \cdot 69^{*}$ & $2 \cdot 04,6 \cdot 67$ & $2 \cdot 61^{*}$ & $1 \cdot 09,6 \cdot 22$ & $4 \cdot 68^{*}$ & $2 \cdot 36,9 \cdot 28$ & $5 \cdot 11^{*}$ & $2 \cdot 53,10 \cdot 32$ & 0.96 & $0.55,1.69$ \\
\hline Methionine§॥ & $1 \cdot 80^{*}$ & $1 \cdot 04,3 \cdot 10$ & 1.58 & $0.69,3.60$ & 1.66 & $0 \cdot 88,3 \cdot 11$ & $2 \cdot 07^{\star}$ & $1 \cdot 12,3 \cdot 81$ & $1 \cdot 30$ & $0 \cdot 75,2 \cdot 22$ \\
\hline Choline§ & $3 \cdot 63$ & $0 \cdot 93,14 \cdot 10$ & $1.66 \S$ & $0 \cdot 72,3 \cdot 83$ & $1 \cdot 05 \S$ & $0.56,1.97$ & $2 \cdot 66$ & $0.55,12 \cdot 81$ & 0.33 & $0.08,1.32$ \\
\hline Betaine§॥ & $6 \cdot 66^{\star}$ & $3.55,12 \cdot 49$ & $2 \cdot 61^{*}$ & $1 \cdot 03,6 \cdot 60$ & $15 \cdot 10^{*}$ & $6 \cdot 88,33 \cdot 13$ & $11 \cdot 44^{*}$ & $5 \cdot 35,24 \cdot 46$ & 1.05 & $0.60,1.85$ \\
\hline \multicolumn{11}{|l|}{ Bone-related } \\
\hline Vitamin D & $2 \cdot 85^{\star}$ & $1 \cdot 45,5 \cdot 59$ & $0 \cdot 35^{\star}$ & $0 \cdot 14,0.87$ & $1 \cdot 60$ & $0 \cdot 74,3 \cdot 50$ & $4 \cdot 49^{\star}$ & $1 \cdot 78,11 \cdot 35$ & $1 \cdot 93^{\star}$ & $1 \cdot 01,3.68$ \\
\hline $\mathrm{Ca}$ & $1 \cdot 59$ & $0 \cdot 76,3 \cdot 32$ & $3 \cdot 15^{\star}$ & $1 \cdot 07,9 \cdot 27$ & $2 \cdot 39$ & $0.97,5 \cdot 90$ & 0.97 & $0 \cdot 42,2 \cdot 23$ & $1 \cdot 54$ & $0 \cdot 75,3 \cdot 18$ \\
\hline $\mathrm{P} \S \|$ & 0.92 & $0.54,1.58$ & $1 \cdot 37$ & $0 \cdot 59,3 \cdot 15$ & 0.92 & $0 \cdot 49,1 \cdot 74$ & $0 \cdot 62$ & $0 \cdot 34,1 \cdot 15$ & $2 \cdot 18^{\star}$ & $1 \cdot 25,3 \cdot 79$ \\
\hline \multicolumn{11}{|l|}{ Electrolytes } \\
\hline $\mathrm{K} \S$ & $1 \cdot 17$ & $0 \cdot 67,2 \cdot 03$ & $2 \cdot 85^{\star}$ & $1 \cdot 19,6 \cdot 81$ & $1 \cdot 64$ & $0 \cdot 86,3 \cdot 13$ & $1 \cdot 15$ & $0 \cdot 62,2 \cdot 11$ & $1 \cdot 11$ & $0.64,1.94$ \\
\hline $\mathrm{Na} \S \|$ & 1.03 & $0 \cdot 60,1 \cdot 78$ & $1 \cdot 40$ & $0 \cdot 61,3 \cdot 19$ & $1 \cdot 68$ & $0 \cdot 89,3 \cdot 16$ & $2 \cdot 15^{\star}$ & $1 \cdot 17,3 \cdot 97$ & 1.53 & $0 \cdot 89,2 \cdot 63$ \\
\hline \multicolumn{11}{|l|}{ Other } \\
\hline Thiamin & 0.93 & $0.30,2 \cdot 93$ & $2 \cdot 52^{\star} \S$ & $1 \cdot 04,6 \cdot 10$ & $4 \cdot 66 * \S$ & $2 \cdot 32,9 \cdot 39$ & 1.54 & $0 \cdot 47,5 \cdot 05$ & $0 \cdot 87$ & $0.29,2.63$ \\
\hline Niacin & $2 \cdot 15$ & $0 \cdot 62,7 \cdot 51$ & $1.98 \S$ & $0 \cdot 83,4.71$ & $3.97 * \S$ & $2 \cdot 01,7 \cdot 86$ & $4 \cdot 43^{\star}$ & $1 \cdot 24,15 \cdot 75$ & 0.98 & $0 \cdot 30,3 \cdot 16$ \\
\hline Vitamin KII & $1 \cdot 38$ & $0 \cdot 67,2 \cdot 82$ & $5 \cdot 67^{*}$ & $1 \cdot 82,17 \cdot 67$ & $3 \cdot 67^{\star}$ & $1 \cdot 35,10 \cdot 03$ & $2 \cdot 06$ & $0.96,4.44$ & $1 \cdot 20$ & $0.58,2 \cdot 48$ \\
\hline
\end{tabular}

PRISM, PRogramming of Intergenerational Stress Mechanisms.

All analyses were adjusted for maternal age, BMI and smoking during pregnancy.

${ }^{*}$ Represents significance $(P<0.05)$.

tWomen born in the USA are the reference group.

fCaucasian women are the reference group. There were no significant findings among the other/mixed race category (data not shown).

$\S$ The median cut-point was used to define high and low total intake (vitamin A: $1133.62 \mu \mathrm{g} ; \beta$-carotene: $4172.63 \mu \mathrm{g}$; Se: $111.36 \mu \mathrm{g}$; Zn: $13.58 \mathrm{mg}$; vitamin B $\mathrm{B}_{12}$ : $6.79 \mu \mathrm{g}$; riboflavin: $2.62 \mathrm{mg}$; methionine: $1.49 \mathrm{mg}$; choline: $311.47 \mathrm{mg}$; betaine: $159.89 \mathrm{mg}$; P: $1352.36 \mathrm{mg}$; K: 2.78 g; Na: $3.15 \mathrm{~g}$; thiamin: $1.95 \mathrm{mg} ;$ niacin: $26 \cdot 33 \mathrm{mg})$.

IIBased on dietary intake only.

and low intakes. African American race $(\mathrm{OR}=2 \cdot 85 ; 95 \%$ CI $1 \cdot 19,6 \cdot 81)$ and low educational status (OR $=2 \cdot 44$; $95 \%$ CI $1.32,4.51)$ were the only correlates of low $\mathrm{K}$ intakes (Tables 3 and 4, respectively). Conversely, the amount of $\mathrm{Na}$ consumed by this cohort was much higher than the AI level of $1.5 \mathrm{~g} / \mathrm{d}$ (mean intake of $3.19 \mathrm{~g} / \mathrm{d}$ ) with 98.9\% consuming more than the $\mathrm{AI}$ and $98.2 \%$ having $\mathrm{Na}$ intakes above the UL of $2 \cdot 3 \mathrm{~g} / \mathrm{d}$. Spanish-speaking women consumed less $\mathrm{Na}$ than those women whose primary language was English $(\mathrm{OR}=2 \cdot 15 ; 95 \% \mathrm{CI} 1 \cdot 17,3 \cdot 97)$.

\section{Intake of other nutrients}

The frequency of thiamin (6.9\%) and niacin (5.8\%) inadequacies was relatively low and did not vary by nativity or ethnic identity (Table 3). However, African Americans and Hispanics and low maternal education were significantly associated with lower intakes of thiamin and niacin (Tables 3 and 4, respectively). Thiamin and niacin inadequacies were also associated with food insecurity $(\mathrm{OR}=4 \cdot 87 ; 95 \% \mathrm{CI} 1 \cdot 61,14 \cdot 78)$ and speaking Spanish $(\mathrm{OR}=4 \cdot 43 ; 95 \%$ CI $1 \cdot 24,15 \cdot 75)$, respectively.
Vitamin K intakes below the AI level were prevalent in over $20 \%$ of the study population (Table 2). Hispanics $(\mathrm{OR}=3 \cdot 67 ; 95 \% \mathrm{CI} 1.35,10.03)$ and African Americans $(\mathrm{OR}=5 \cdot 67 ; 95 \%$ CI $1 \cdot 82,17 \cdot 67)$ were more likely to have lower vitamin $\mathrm{K}$ intake than Caucasians. Low vitamin $\mathrm{K}$ intake was significantly more common among women with a lower level of education or more difficulty living on their total family income or meeting monthly payments/ bills (Table 4).

\section{Discussion}

These analyses, considering both dietary and supplemental intakes, identify a range of micronutrient intake inadequacies in a multi-ethnic, primarily urban US population of pregnant women that may have implications for perinatal and more long-term child health. These analyses expand prior research involving pregnant US samples that pre-dated changes in folic acid awareness and its widespread fortification or more narrowly considered 
Table 4 Associations between food insecurity and sociodemographic factors and total (dietary + supplement) micronutrient inadequacy in the study participants: pregnant women ( $n$ 274), PRISM study, Boston, MA, USA, March 2011-August 2012

\begin{tabular}{|c|c|c|c|c|c|c|c|c|}
\hline \multirow[b]{2}{*}{ Micronutrients } & \multicolumn{2}{|c|}{ Low maternal educationt } & \multicolumn{2}{|c|}{ Food insecurity } & \multicolumn{2}{|c|}{ Difficulty with incomeł } & \multicolumn{2}{|c|}{ Difficulty with billsł } \\
\hline & OR & $95 \% \mathrm{Cl}$ & OR & $95 \% \mathrm{Cl}$ & OR & $95 \% \mathrm{Cl}$ & OR & $95 \% \mathrm{Cl}$ \\
\hline \multicolumn{9}{|l|}{ Antioxidants } \\
\hline Vitamin A & $4 \cdot 14^{*}$ & $1 \cdot 54,11 \cdot 11$ & $2 \cdot 64^{*}$ & $1 \cdot 04,6 \cdot 67$ & $2 \cdot 46$ & $0 \cdot 77,7 \cdot 84$ & $1 \cdot 49$ & $0.55,4.03$ \\
\hline$\beta$-Carotene $\S$ & $3 \cdot 71$ & $1 \cdot 96,7 \cdot 01$ & $1 \cdot 81$ & $0.98,3.37$ & $3 \cdot 11^{*}$ & $1 \cdot 73,5 \cdot 56$ & $3 \cdot 60^{*}$ & $2 \cdot 01,6 \cdot 43$ \\
\hline Vitamin C & $4 \cdot 85^{\star}$ & $1 \cdot 62,14 \cdot 52$ & $6 \cdot 09^{\star}$ & $2 \cdot 16,17 \cdot 16$ & $4 \cdot 20^{*}$ & $1 \cdot 14,15 \cdot 56$ & $4 \cdot 37^{*}$ & $1 \cdot 31,15 \cdot 50$ \\
\hline Vitamin E & $4 \cdot 08^{*}$ & $2 \cdot 13,7 \cdot 81$ & $2 \cdot 53^{\star}$ & $1 \cdot 33,4 \cdot 83$ & $2 \cdot 29^{\star}$ & $1 \cdot 29,4 \cdot 05$ & $2 \cdot 26^{*}$ & $1 \cdot 29,3 \cdot 98$ \\
\hline $\mathrm{Mg}$ & $2 \cdot 19^{*}$ & $1 \cdot 19,4 \cdot 04$ & $1 \cdot 92^{*}$ & $1 \cdot 03,3 \cdot 56$ & $1 \cdot 42$ & $0.79,2.53$ & $1 \cdot 24$ & $0 \cdot 70,2 \cdot 20$ \\
\hline Seß & $3 \cdot 12^{*}$ & $1 \cdot 68,5 \cdot 80$ & $2 \cdot 57^{\star}$ & $1 \cdot 37,4 \cdot 82$ & $1 \cdot 62$ & $0.93,2 \cdot 81$ & $1 \cdot 75^{*}$ & $1 \cdot 01,3 \cdot 02$ \\
\hline $\mathrm{Zn}$ & $3 \cdot 24^{*}$ & $1 \cdot 40,7 \cdot 54$ & $1 \cdot 45$ & $0 \cdot 64,3 \cdot 31$ & 1.93 & $0 \cdot 76,4 \cdot 88$ & $1 \cdot 55$ & $0.66,3.65$ \\
\hline \multicolumn{9}{|c|}{ Methyl/epigenetic nutrients } \\
\hline Vitamin $\mathrm{B}_{12}$ & $1 \cdot 22$ & $0 \cdot 22,6 \cdot 71$ & $0 \cdot 84$ & $0 \cdot 15,4 \cdot 72$ & $1 \cdot 05$ & $0 \cdot 18,6 \cdot 16$ & $0 \cdot 71$ & $0 \cdot 14,3 \cdot 64$ \\
\hline Vitamin $\mathrm{B}_{6}$ & $3 \cdot 39^{*}$ & $1 \cdot 51,7 \cdot 62$ & 1.40 & $0.63,3 \cdot 14$ & $1 \cdot 73$ & $0 \cdot 72,4 \cdot 19$ & $1 \cdot 44$ & $0.63,3.28$ \\
\hline Folate & 0.93 & $0 \cdot 40,2 \cdot 16$ & $1 \cdot 68$ & $0 \cdot 77,3 \cdot 70$ & $3 \cdot 22^{*}$ & $1 \cdot 24,8 \cdot 35$ & $2 \cdot 14$ & $0.93,4.91$ \\
\hline Riboflavin & $2 \cdot 73$ & $0 \cdot 46,16 \cdot 36$ & $0 \cdot 84$ & $0 \cdot 15,4 \cdot 71$ & $1 \cdot 07$ & $0 \cdot 18,6 \cdot 21$ & $0 \cdot 72$ & $0 \cdot 14,3.68$ \\
\hline $\mathrm{Fe}$ & $3 \cdot 33^{*}$ & $1 \cdot 74,6 \cdot 39$ & $2 \cdot 31^{\star}$ & $1 \cdot 20,4 \cdot 45$ & $2 \cdot 09^{*}$ & $1 \cdot 19,3 \cdot 65$ & $2 \cdot 31^{*}$ & $1 \cdot 32,4.05$ \\
\hline Methionine§̧॥ & $1 \cdot 63$ & $0.90,2.95$ & $1 \cdot 00$ & $0.55,1 \cdot 82$ & 0.93 & $0.54,1.60$ & $0 \cdot 86$ & $0.50,1 \cdot 47$ \\
\hline Cholinell & 0.46 & $0.14,1.56$ & 0.37 & $0 \cdot 11,1 \cdot 19$ & 0.38 & $0 \cdot 10,1 \cdot 48$ & $0 \cdot 40$ & $0 \cdot 11,1 \cdot 41$ \\
\hline Betaine§॥ & $7 \cdot 60^{*}$ & $3 \cdot 80,15 \cdot 20$ & $3 \cdot 26^{*}$ & $1 \cdot 69,6 \cdot 27$ & $3 \cdot 11^{*}$ & $1 \cdot 73,5 \cdot 56$ & $3 \cdot 60^{*}$ & $2 \cdot 01,6 \cdot 43$ \\
\hline \multicolumn{9}{|l|}{ Bone-related } \\
\hline Vitamin D & $2 \cdot 48^{*}$ & $1 \cdot 14,5 \cdot 42$ & $1 \cdot 24$ & $0.59,2.58$ & 1.05 & $0.56,1.98$ & $1 \cdot 36$ & $0.72,2.57$ \\
\hline $\mathrm{Ca}$ & 1.51 & $0 \cdot 71,3 \cdot 21$ & $1 \cdot 61$ & $0 \cdot 77,3 \cdot 36$ & $1 \cdot 48$ & $0.71,3.08$ & $1 \cdot 52$ & $0 \cdot 74,3 \cdot 13$ \\
\hline$P \S \|$ & $0 \cdot 70$ & $0.39,1.27$ & $1 \cdot 11$ & $0.61,2 \cdot 04$ & 0.78 & $0.45,1.35$ & 0.69 & $0 \cdot 40,1 \cdot 20$ \\
\hline \multicolumn{9}{|l|}{ Electrolytes } \\
\hline $\mathrm{K} \S \|$ & $2 \cdot 44^{*}$ & $1 \cdot 32,4 \cdot 51$ & $1 \cdot 25$ & $0 \cdot 68,2 \cdot 31$ & $1 \cdot 06$ & $0 \cdot 61,1 \cdot 83$ & $1 \cdot 06$ & $0 \cdot 61,1 \cdot 84$ \\
\hline $\mathrm{Na} \S \|$ & $1 \cdot 26$ & $0 \cdot 70,2 \cdot 26$ & $1 \cdot 24$ & $0 \cdot 68,2 \cdot 25$ & $1 \cdot 11$ & $0.65,1.91$ & $1 \cdot 16$ & $0.68,1.99$ \\
\hline \multicolumn{9}{|l|}{ Other } \\
\hline Thiamin & $8 \cdot 20^{*}$ & $2 \cdot 24,30 \cdot 00$ & $4 \cdot 87^{\star}$ & $1 \cdot 61,14 \cdot 78$ & $2 \cdot 22$ & $0.58,8.55$ & $3 \cdot 30$ & $0 \cdot 86,12 \cdot 71$ \\
\hline Niacin & $6 \cdot 56^{*}$ & $1 \cdot 64,26 \cdot 20$ & $2 \cdot 55$ & $0.82,7.92$ & $2 \cdot 02$ & $0.52,7.91$ & $5 \cdot 20^{*}$ & $1 \cdot 08,25 \cdot 07$ \\
\hline Vitamin KII & $3 \cdot 16^{*}$ & $1 \cdot 53,6 \cdot 54$ & $1 \cdot 86$ & $0.91,3.84$ & $4 \cdot 01^{*}$ & $1 \cdot 66,9 \cdot 72$ & $2 \cdot 29^{*}$ & $1 \cdot 08,4 \cdot 85$ \\
\hline
\end{tabular}

PRISM, PRogramming of Intergenerational Stress Mechanisms.

All analyses were adjusted for maternal age, BMI and smoking during pregnancy.

${ }^{*}$ Represents significance $(P<0.05)$.

+Women with some college education or college degree are the reference group.

$\ddagger$ Women with no difficulty are the reference group.

$\S$ The median cut-point was used to define high and low intake ( $\beta$-carotene: $4172.63 \mu \mathrm{g}$; Se: $111 \cdot 36 \mu \mathrm{g}$; methionine: $1.49 \mathrm{mg}$; betaine: $159 \cdot 89 \mathrm{mg}$; P: $1352 \cdot 36 \mathrm{mg} ; \mathrm{K}: 2 \cdot 78 \mathrm{~g} ; \mathrm{Na}: 3 \cdot 15 \mathrm{~g})$;

IIBased on dietary intake only.

multivitamin use. We also identified vulnerable groups of women with distinct differences in dietary micronutrient composition related to sociodemographic factors that may inform tailored intervention strategies going forward.

Of the antioxidant inadequacies identified, vitamin $\mathrm{E}$ and $\mathrm{Mg}$ were most striking. Notably, the EAR for vitamin E is the same $(12 \mathrm{mg} / \mathrm{d})$ in pregnant and non-pregnant women; $\mathrm{Mg}$ requirements increase slightly during pregnancy $(255 \mathrm{mg} / \mathrm{d}$ preconception to $290 \mathrm{mg} / \mathrm{d}$ in pregnancy). Although Mg intake exceeded the UL in some women, the prevalence of intake below the EAR was over $30 \%$. Hispanics and African Americans were more likely to report antioxidant inadequacies compared with Caucasians, with the exception of Mg. African Americans were most likely to have inadequate vitamin E intake. As other studies suggest that African Americans are less likely to select from food groups rich in $\alpha$-tocopherol and $\mathrm{Mg}$, such as nuts, seeds, whole grains, green leafy vegetables and vegetable oils $^{(47)}$, this may represent a unique target for dietary intervention among this group. A recent pilot study demonstrated the feasibility and acceptability of a food-exchange-based intervention to optimize prenatal dietary vitamin $\mathrm{E}$ intake ${ }^{(48)}$. Such studies should be expanded to include ethnic minority, urban populations.

Nearly all women reported choline intakes lower than the recommended AI. This is concerning since choline plays an important role in placental function and fetal development (birth defects, brain development), possibly by influencing DNA methylation status ${ }^{(8,49)}$. As prenatal vitamins do not contain an adequate source of choline, some have proposed that women with low intakes of milk, meat, eggs or other choline-rich foods should consider a prenatal dietary supplement containing this micronutrient. Similar to findings in a number of developed countries (e.g. Japan, Australia, Europe, UK) ${ }^{(12)}$, Fe inadequacy, which may also influence epigenetic modifications ${ }^{(50-52)}$, was common in these women. Pregnant women are particularly vulnerable to Fe deficiency given that the EAR for Fe increases by approximately $15 \mathrm{mg} / \mathrm{d}$ in pregnancy $^{(34)}$. However, even after considering supplementation in our sample, $57 \%$ of women had inadequate Fe intakes and reported insufficient intakes of relevant food sources, which include red meats, fish, poultry, beans, lentils and fortified foods ${ }^{(53)}$. African American, 
Hispanic and foreign-born women consumed less $\mathrm{Fe}$ and other methyl nutrients, including vitamin $\mathrm{B}_{6}$ and betaine, compared with Caucasians and US-born women, respectively. While disparities in Fe intake among pregnant African Americans and Hispanics have been reported ${ }^{(54)}$, nativity status has been less well studied. One study found that pregnant Hispanic immigrants consumed more folate, vitamin $\mathrm{C}, \mathrm{Fe}$ and $\mathrm{Zn}$ than US-born Hispanics ${ }^{(55)}$. Longer duration of US residence has been attributed to lower energy intake ${ }^{(21)}$. Our study showed decreases in antioxidant and methyl-nutrient intakes among Hispanics and foreign-born women. Dissimilarities in micronutrient intakes by race and nativity could partly be explained by differences in the use of supplements, as previously shown with $\mathrm{Fe}^{(56)}$. The complex relationships among race, nativity status and prenatal micronutrient intakes warrant further investigation.

Inadequate vitamin D intake was present in over $75 \%$ of these women from the north-eastern USA, where vitamin D status is also likely influenced by season given the importance of sun exposure and consequent conversion to 25-hydroxyvitamin $D^{(57)}$. Even moderately decreased levels of 25-hydroxyvitamin $\mathrm{D}$ have been associated with poor fetal and infant skeletal growth and tooth mineralization ${ }^{(58)}$. Therefore, inadequate dietary intake of vitamin $\mathrm{D}$ may be more relevant in populations, such as this cohort, affected by seasonal climates where sun exposure, as a source of vitamin $\mathrm{D}$, is limited. In our study, foreign-born women and those with low ethnic identity were most likely to have bone-related micronutrient inadequacies. Immigrant groups perceiving that their American identity is being challenged $^{(59)}$ may abandon their ethnic identity and conform to an American identity, in part, by embracing poor dietary behaviours $^{(60)}$. Thus, ethnic identity may be another important factor to consider when identifying vulnerable populations at risk for suboptimal nutrient status.

Low maternal education consistently predicted inadequate antioxidant and suboptimal or inadequate methylnutrient intakes. Data from non-pregnant US samples similarly report that education is an important predictor of diet quality ${ }^{(19)}$. When micronutrient intakes, including vitamins $\mathrm{A}, \mathrm{C}, \mathrm{D}, \mathrm{E}$ and $\mathrm{B}_{12}, \beta$-carotene, folate, $\mathrm{Fe}$, Ca and $\mathrm{Mg}$, were examined in relation to diet cost and SES (indexed by education and income), researchers found that lower-cost/quality diets were also consumed more frequently by lower-SES groups in the USA ${ }^{(18)}$. Taken together, these findings suggest that both maternal education and SES play a role in dietary intake.

Food insecurity was also significantly associated with antioxidant inadequacies (except $\mathrm{Zn}$ and $\beta$-carotene) and the methyl nutrients $\mathrm{Fe}$ and betaine. Food insecurity, characterized by limited and/or uncertain availability or access to nutritionally adequate foods ${ }^{(61)}$, affected nearly $30 \%$ of this population. Some have proposed that food insecurity could impact fetal development through physiological and psychological changes due to micronutrient deficiencies or the stress experienced as a result of limited access to nutritionally adequate food, respectively ${ }^{(62)}$. US and Canadian studies suggest that individuals experiencing food insecurity have lower intakes of fruits and vegetables ${ }^{(20,63,64)}$, in part due to the perception that nutrientdense food groups are expensive ${ }^{(65)}$. Our findings highlight the need for an enhanced elucidation of the impact of food insecurity on micronutrient intakes in multi-ethnic US pregnant women.

It is well established that not only nutrient deficiencies but also excesses can have adverse effects on perinatal outcomes and fetal development ${ }^{(6)}$. As the majority of pregnant women in the USA take a dietary supplement, most frequently a multivitamin/mineral product, they may be more likely to exceed the UL. When considering food intakes combined with supplements, more than $35 \%$ of the women in the present study reported $\mathrm{Mg}$, folate and niacin intakes higher than the UL. In addition, nearly the entire study population (98\%) had $\mathrm{Na}$ intakes above the UL. Women reporting excessive intakes of $\mathrm{Mg}$, folate and niacin were more likely to be Caucasian or Hispanic, of higher SES, US-born and not reporting food insecurity. These findings highlight the importance of including supplement use when examining dietary intakes during pregnancy and identifying excessive intakes as well as inadequacies.

Interpretation of our findings should be considered in light of our study's limitations. Food and supplemental intakes were based on participant recall and therefore subjected to under- or overestimation. For example, the multiple food items and choices in an FFQ may result in overestimation for some nutrients, and the retrospective nature of the questionnaire may influence recall. However, FFQ are useful for obtaining estimates of usual intake over time (e.g. months), including during pregnancy ${ }^{(12)}$. Misclassification of nutrient intake is likely random and therefore would be expected to result in an underestimation of associations. Nevertheless, future research would be enhanced through the incorporation of food records and $24 \mathrm{~h}$ dietary recalls. Second, the median was used as the cut-point to define high and low micronutrient intakes when an EAR or AI was not available. This may not represent a true inadequacy. Thus, the results are best viewed as estimates rather than absolute levels of intake because FFQ may contain measurement error. We acknowledge that the assessment of food insecurity was not comprehensive. Nevertheless, our findings concerning economically related food insecurity are interesting and warrant further investigation. The approximately $70 \%$ rate of participation at enrolment may reduce generalizability; however, women who were eligible and agreed to participate did not differ on key covariates when compared with those who declined.

\section{Conclusions}

The present study provides a thorough examination of the influence of race/ethnicity, SES and other sociodemographic factors, including nativity status and ethnic 
identity, on micronutrient intakes in a sociodemographically diverse sample of pregnant women in the USA. These data highlight the high prevalence of inadequate intakes of specific micronutrients (e.g. antioxidants, methyl nutrients, electrolytes, bone-related micronutrients) that have been implicated in the prenatal programming of developmental diseases, including allergies and asthma ${ }^{(67)}$, obesity $^{(68)}$ and neurodevelopment ${ }^{(3)}$. Moreover, in utero micronutrient inadequacies may enhance fetal vulnerability to other environmental exposures (e.g. air pollution, stress $)^{(3,69)}$. Prenatal nutritional interventions may be enhanced in urban ethnic-minority populations if tailored to account for the specific sociodemographic determinants of prenatal dietary patterns.

\section{Acknowledgements}

Sources of funding: This work was supported by the National Institute of Environmental Health Sciences (NIEHS; grant number R21ES021318-01). NIEHS had no role in the design, analysis or writing of this article. Conflicts of interest: The authors report no conflict of interest. Authors' contributions: R.J.W. and S.K. are co-senior authors. K.J.B. drafted the manuscript, contributed to the concept and design of the analyses, and led the analyses, interpretation of data and writing of the manuscript; R.O.W. contributed significantly to analyses and interpretation of data and provided critical review and revisions of the manuscript; K.D. contributed to the interpretation of data analyses and provided critical review and revisions of the manuscript; M.B.E. contributed to the interpretation of data analyses and provided critical review and revisions of the manuscript; H.F. contributed to the interpretation of data analyses and provided critical review and revisions of the manuscript; R.J.W. contributed to conception and design of the cohort, data acquisition, analyses and interpretation of data, and provided critical review and revisions of the manuscript; S.K. contributed to dietary data acquisition, analyses and interpretation of data, and provided critical review and revisions of the manuscript.

\section{References}

1. Barker DJ, Osmond C, Kajantie E et al. (2009) Growth and chronic disease: findings in the Helsinki Birth Cohort. Ann Hum Biol 36, 445-458.

2. Barker DJP (1998) Mothers, Babies, and Health in Later Life, 2nd ed. Edinburgh: Churchill Livingstone.

3. Monk C, Georgieff MK \& Osterholm EA (2013) Research review: maternal prenatal distress and poor nutrition mutually influencing risk factors affecting infant neurocognitive development. J Child Psychol Psychiatry 54, 115-130.

4. National Advisory Environmental Health Sciences Council (2010) Dietary influences on the human health effects of environmental exposures. http://www.niehs.nih.gov/about/ boards/naehsc/agenda/may2010/dietary_influences_on_the_ human_health_effects_of_environmental_exposures.pdf (accessed May 2013).
5. Baccarelli A, Cassano PA, Litonjua A et al. (2008) Cardiac autonomic dysfunction: effects from particulate air pollution and protection by dietary methyl nutrients and metabolic polymorphisms. Circulation 117, 1802-1809.

6. Romieu I, Castro-Giner F, Kunzli N et al. (2008) Air pollution, oxidative stress and dietary supplementation: a review. Eur Res J 31, 179-197.

7. Gallo C, Renzi P, Loizzo S et al. (2010) Potential therapeutic effects of vitamin $\mathrm{E}$ and $\mathrm{C}$ on placental oxidative stress induced by nicotine: an in vitro evidence. Open Biochem J 4, 77-82.

8. Jiang X, Yan J, West AA et al. (2012) Maternal choline intake alters the epigenetic state of fetal cortisol-regulating genes in humans. FASEB J 26, 3563-3574.

9. Baibazarova E, van de Beek C, Cohen-Kettenis PT et al. (2013) Influence of prenatal maternal stress, maternal plasma cortisol and cortisol in the amniotic fluid on birth outcomes and child temperament at 3 months. Psychoneuroendocrinology 38, 907-915.

10. Wright RJ, Fisher K, Chiu YH et al. (2013) Disrupted prenatal maternal cortisol, maternal obesity, and childhood wheeze: insights into prenatal programming. Am J Respir Crit Care Med 187, 1186-1193.

11. Tulchinsky TH (2010) Micronutrient deficiency conditions: global health issues. Public Health Rev 32, 12.

12. Blumfield ML, Hure AJ, Macdonald-Wicks L et al. (2013) A systematic review and meta-analysis of micronutrient intakes during pregnancy in developed countries. Nutr Rev 71, 118-132.

13. Turner RE, Langkamp-Henken B, Littell RC et al. (2003) Comparing nutrient intake from food to the estimated average requirements shows middle- to upper-income pregnant women lack iron and possibly magnesium. J Am Diet Assoc 103, 461-466.

14. Lenders CM, Hediger ML, Scholl TO et al. (1997) Gestational age and infant size at birth are associated with dietary sugar intake among pregnant adolescents. $J$ Nutr 127, 1113-1117.

15. Rifas-Shiman SL, Rich-Edwards JW, Willett WC et al. (2006) Changes in dietary intake from the first to the second trimester of pregnancy. Paediatr Perinat Epidemiol 20, $35-42$.

16. Swensen AR, Harnack LJ \& Ross JA (2001) Nutritional assessment of pregnant women enrolled in the Special Supplemental Program for Women, Infants, and Children (WIC). J Am Diet Assoc 101, 903-908.

17. Vaccaro JA \& Huffman FG (2013) Race/ethnicity-, genderand age-specific differences in micronutrient intakes of US adults with and without diabetes. Int J Food Sci Nutr 64, 175-184

18. Aggarwal A, Monsivais P \& Drewnowski A (2012) Nutrient intakes linked to better health outcomes are associated with higher diet costs in the US. PLoS One 7, e37533.

19. Kant AK \& Graubard BI (2007) Secular trends in the association of socio-economic position with self-reported dietary attributes and biomarkers in the US population: National Health and Nutrition Examination Survey (NHANES) 1971-1975 to NHANES 1999-2002. Public Health Nutr 10, 158-167.

20. Kirkpatrick SI \& Tarasuk V (2008) Food insecurity is associated with nutrient inadequacies among Canadian adults and adolescents. J Nutr 138, 604-612.

21. Harley K, Eskenazi B \& Block G (2005) The association of time in the US and diet during pregnancy in low-income women of Mexican descent. Paediatr Perinat Epidemiol 19, 125-134.

22. Perez-Escamilla R (2011) Acculturation, nutrition, and health disparities in Latinos. Am J Clin Nutr 93, issue 5, 1163S-1167S. 
23. Langley-Evans SC (2013) Fetal programming of CVD and renal disease: animal models and mechanistic considerations. Proc Nutr Soc 72, 317-325.

24. Block G, Hartman AM, Dresser CM et al. (1986) A databased approach to diet questionnaire design and testing. Am J Epidemiol 124, 453-469.

25. Snook Parrott M, Bodnar LM, Simhan HN et al. (2009) Maternal cereal consumption and adequacy of micronutrient intake in the periconceptional period. Public Health Nutr 12, 1276-1283.

26. Volgyi E, Carroll KN, Hare ME et al. (2013) Dietary patterns in pregnancy and effects on nutrient intake in the Mid-South: the Conditions Affecting Neurocognitive Development and Learning in Early Childhood (CANDLE) Study. Nutrients $\mathbf{5}$, 1511-1530.

27. Siega-Riz AM, Bodnar LM \& Savitz DA (2002) What are pregnant women eating? Nutrient and food group differences by race. Am J Obstet Gynecol 186, 480-486.

28. US Department of Agriculture, Nutrient Data Laboratory (1998) USDA National Nutrient Database for Standard Reference. http://www.ars.usda.gov/nutrientdata (accessed January 2013).

29. Institute of Medicine (2000) Dietary Reference Intakes for Thiamin, Riboflavin, Niacin, Vitamin $B_{6}$, Folate, Vitamin $B_{12}$, Pantothenic Acid, Biotin, and Choline. Washington, DC: The National Academies Press.

30. US Department of Agriculture, Beltsville Human Nutrition Research Center (2004) USDA Database for the Choline Content of Common Foods. http://www.algry.com/ing/6/ doc/08.pdf (accessed May 2013).

31. US Department of Agriculture, Beltsville Human Nutrition Research Center (2008) USDA Database for the Choline Content of Common Foods, Release 2. http://www.ars.usda. gov/SP2UserFiles/Place/12354500/Data/Choline/Choln02.pdf (accessed May 2013).

32. Institute of Medicine (1997) Dietary Reference Intakes for Calcium, Phosphorus, Magnesium, Vitamin D, and Fluoride. Washington, DC: The National Academies Press.

33. Institute of Medicine (2000) Dietary Reference Intakes for Vitamin C, Vitamin E, Selenium, and Carotenoids. Washington, DC: The National Academies Press.

34. Institute of Medicine (2001) Dietary Reference Intakes for Vitamin A, Vitamin K, Arsenic, Boron, Chromium, Copper, Iodine, Iron, Manganese, Molybdenum, Nickel, Silicon, Vanadium, and Zinc. Washington, DC: The National Academies Press.

35. Institute of Medicine (2002) Dietary Reference Intakes for Energy, Carbohydrate, Fiber, Fat, Fatty Acids, Cholesterol, Protein, and Amino Acids. Washington, DC: The National Academies Press.

36. Institute of Medicine (2011) Dietary Reference Intakes for Calcium and Vitamin D. Washington, DC: The National Academies Press.

37. Meltzer HM, Brantsaeter AL, Ydersbond TA et al. (2008) Methodological challenges when monitoring the diet of pregnant women in a large study: experiences from the Norwegian Mother and Child Cohort Study (MoBa). Matern Child Nutr 4, 14-27.

38. Pearlin LI, Lieberman MA, Menaghan EG et al. (1981) The stress process. J Health Soc Behav 22, 337-356.

39. World Health Organization (2006) Global database on BMI classification. http://www.who.int/bmi/index.Jsp?Intropageintro_3.html (accessed May 2013).

40. Phinney JS (1992) The Multigroup Ethnic Identity Measure: a new scale for use with diverse groups. J Adolesc Res 7, 20.

41. Nguyen KH, Subramanian SV, Sorensen G et al. (2012) Influence of experiences of racial discrimination and ethnic identity on prenatal smoking among urban black and Hispanic women. J Epidemiol Community Health 66, 315-321.
42. Shalowitz MU, Berry CA, Rasinski KA et al. (1998) A new measure of contemporary life stress: development, validation, and reliability of the CRISYS. Health Serv Res 33, 1381-1402.

43. Willett WC, Howe GR \& Kushi LH (1997) Adjustment for total energy intake in epidemiological studies. Am J Clin Nutr 65, 4 Suppl., 1220S-1228S.

44. Cuco G, Fernandez-Ballart J, Sala J et al. (2006) Dietary patterns and associated lifestyles in preconception, pregnancy and postpartum. Eur J Clin Nutr 60, 364-371.

45. Olinto MT, Willett WC, Gigante DP et al. (2011) Sociodemographic and lifestyle characteristics in relation to dietary patterns among young Brazilian adults. Public Health Nutr 14, 150-159.

46. Padrao P, Silva-Matos C, Damasceno A et al. (2011) Association between tobacco consumption and alcohol, vegetable and fruit intake across urban and rural areas in Mozambique. J Epidemiol Community Health 65, 445-453.

47. Kant AK, Graubard BI \& Kumanyika SK (2007) Trends in black-white differentials in dietary intakes of US adults, 1971-2002. Am J Prev Med 32, 264-272.

48. Clark J, Craig L, McNeill G et al. (2012) A novel dietary intervention to optimize vitamin $\mathrm{E}$ intake of pregnant women to $15 \mathrm{mg}$ /day. J Acad Nutr Diet 112, 297-301.

49. Boeke CE, Baccarelli A, Kleinman KP et al. (2012) Gestational intake of methyl donors and global LINE-1 DNA methylation in maternal and cord blood: prospective results from a folate-replete population. Epigenetics 7 , $253-260$

50. Cyr AR \& Domann FE (2011) The redox basis of epigenetic modifications: from mechanisms to functional consequences. Antioxid Redox Signal 15, 551-589.

51. Yi CQ, Yang CG \& He C (2009) A non-heme iron-mediated chemical demethylation in DNA and RNA. Acc Chem Res 42, 519-529.

52. Zhang HK, Zhang X, Clark E et al. (2010) TET1 is a DNAbinding protein that modulates DNA methylation and gene transcription via hydroxylation of 5-methylcytosine. Cell Res 20, 1390-1393.

53. Office of Dietary Supplements \& National Center for Complementary and Alternative Medicine, National Institutes of Health (2013) Home page. http://ods.od.nih.gov/ (accessed January 2013).

54. Mei Z, Cogswell ME, Looker AC et al. (2011) Assessment of iron status in US pregnant women from the National Health and Nutrition Examination Survey (NHANES), 1999-2006. Am J Clin Nutr 93, 1312-1320.

55. Schaffer DM, Velie EM, Shaw GM et al. (1998) Energy and nutrient intakes and health practices of Latinas and white non-Latinas in the 3 months before pregnancy. J Am Diet Assoc 98, 876-884.

56. Cogswell ME, Parvanta I, Ickes L et al. (2003) Iron supplementation during pregnancy, anemia, and birth weight: a randomized controlled trial. Am J Clin Nutr $\mathbf{7 8 ,}$ 773-781.

57. Wuertz C, Gilbert P, Baier W et al. (2013) Cross-sectional study of factors that influence the 25-hydroxyvitamin D status in pregnant women and in cord blood in Germany. BrJ Nutr 110, 1895-1902.

58. Marshall I, Mehta R \& Petrova A (2013) Vitamin D in the maternal-fetal-neonatal interface: clinical implications and requirements for supplementation. J Matern Fetal Neonatal Med 26, 633-638.

59. Cheryan S \& Monin B (2005) 'Where are you really from?': Asian Americans and identity denial. J Pers Soc Psychol 89, 717-730.

60. Guendelman MD, Cheryan S \& Monin B (2011) Fitting in but getting fat: identity threat and dietary choices among US immigrant groups. Psychol Sci 22, 959-967. 
61. Kendall A \& Kennedy E (1998) Position of the American Dietetic Association: domestic food and nutrition security. I Am Diet Assoc 98, 337-342.

62. Ramsey R, Giskes K, Turrell G et al. (2012) Food insecurity among adults residing in disadvantaged urban areas: potential health and dietary consequences. Public Health Nutr 15, 227-237.

63. Kaiser LL, Melgar-Quinonez H, Townsend MS et al. (2003) Food insecurity and food supplies in Latino households with young children. J Nutr Educ Behav 35, 148-153.

64. Tingay RS, Tan CJ, Tan NC et al. (2003) Food insecurity and low income in an English inner city. $J$ Public Health Med 25, 156-159.

65. Tanumihardjo SA, Anderson C, Kaufer-Horwitz M et al. (2007) Poverty, obesity, and malnutrition: an international perspective recognizing the paradox. J Am Diet Assoc 107, 1966-1972.
66. Mao C, Liu R, Li S et al. (2013) High salt diets during pregnancy affected fetal and offspring renal renin-angiotensin system. J Endocrinol 218, 61-73.

67. Erkkola M, Nwaru BI, Kaila M et al. (2012) Risk of asthma and allergic outcomes in the offspring in relation to maternal food consumption during pregnancy: a Finnish birth cohort study. Pediatr Allergy Immunol 23, 186-194.

68. Yang Z \& Huffman SL (2013) Nutrition in pregnancy and early childhood and associations with obesity in developing countries. Matern Child Nutr 9, Suppl. 1, 105-119.

69. Kannan S, Misra DP, Dvonch JT et al. (2006) Exposures to airborne particulate matter and adverse perinatal outcomes: a biologically plausible mechanistic framework for exploring potential effect modification by nutrition. Environ Health Perspect 114, 1636-1642. 